\title{
Seismic Response Analysis of Medium Pressure Vertical Multilayer Pipe Support System in High-rise Building
}

\author{
Ge Cui ${ }^{1, a}$, Hehui Wang ${ }^{2, b}$, Chenqiong Ye ${ }^{1, c}$, Xueyi Yin ${ }^{1, d}$, Bo Liu ${ }^{2, e}$ \\ ${ }^{1}$ General Construction Company of CCTEB Group Co.,Ltd, Wuhan, 430064,China; \\ ${ }^{2}$ School of Mechanical and Power Engineering, East China University of Science and Technology, \\ Shanghai, 200237, China \\ acuige@126.com, bhhwang@ecust.edu.cn, ycq59642502@163.com, d125147589@qq.com, \\ e13122006197@163.com
}

\begin{abstract}
Keywords: fixing trestle; spectrum analysis; seismic evaluation
Abstract: Based on finite element analysis software ANSYS, using shell elements and space beam elements, finite element model of the vertical multilayer pipe support system of high-rise building is created. The dynamic characteristics and seismic response of piping system are analyzed under earthquake load using multi-point earthquake response spectrum analysis, and the strength and stiffness of components of plate and shell are assessed based on the corresponding seismic code. With the superposition calculation of spectrum analysis and static analysis, the results indicate that the strength and stiffness of fixing trestles and pipes satisfy the requirements in the design of the earthquake.
\end{abstract}

\section{Introduction}

With the development of high-rise building, the dynamic characteristics and seismic response analysis and seismic design of the high-rise building pipeline system have become an important issue in engineering design. Under the earthquake, the layout and design of the multilayer pipe support system can effectively affect limit the displacement and vibration of the pipeline, so as to reduce the risks of the overall vibration and pipeline breaks. However, most of the current seismic research in China is only concerned about the building itself, which is less related to finite element analysis of the single pipe support system under earthquake load. In this paper, the seismic response of partial multilayer pipe support system of a high-rise building of Tianjin was analyzed, which contained static analysis, modal analysis, seismic load analysis, as well as the assessment of anti-seismic strength and stiffness of the fixing trestle.

The pipeline support system mainly contains pipes and the support part, which including three pipes, trestles and bearing beams. The pipe section is defined as the pipelines deployed from the third floor $(14.6 \mathrm{~m})$ to the thirteenth floor $(70.77 \mathrm{~m})$, which the pipeline is characterized by large diameter, high pressure, and long span. The diameter of the pipeline is DN700mm, the thickness is $16 \mathrm{~mm}$ and the working pressure is $2.5 \mathrm{MPa}$. The top end of the pipes is designed with corrugated pipe expansion joints that can compensate for its thermal elongation and migration, so that can prevent deformation and failure of pipes due to the thermal stress caused by the medium temperature fluctuation.

\section{Method and model}

Analysis theory. Currently, there are three main methods of seismic response analysis: static coefficient method, response spectrum method and dynamic analysis method. The dynamic analysis specifically contained time-history analysis method and spectral analysis method. The spectrum analysis method is applied in actual seismic response analysis, which is a kind of method based on mode superposition of the linear system ${ }^{[1]}$.

This method is widely used in the design of engineering seismic analysis due to the simple calculation principle and mature theory. The basic principle of response spectrum analysis is that the natural frequencies $\omega_{i}$ and the corresponding modal vectors $\left\{\Phi_{i}\right\}$ of the different frequencies for a $n$-degree 
freedom system will be obtained by modal analysis, and the acceleration response spectrum of the direction of $j(j=x, y, z)$ is $S_{a j}(\omega)$, then the displacement response of the $i$-degree will satisfy Eq. 1

$$
u_{j i}=\left\{\Phi_{i}\right\} \gamma_{i}\left[S_{a j}\left(\omega_{i}\right)\right] / \omega_{i}^{2}
$$

The displacement response of the three directions $(x, y, z)$ can be calculated by the Eq.1, and the system response can be calculated by the square root of the sum of the squares (SRSS) method ${ }^{[2]}$.

Finite element model. Based on the response spectrum analysis function of ANSYS, seismic performance of multi point response spectrum of the multilayer pipe support system was analyzed in this paper, which includes modal analysis, response spectrum analysis, modal expansion and modal merging. Three-dimensional finite element model of the high rise building was established, using shell element and space beam element. By applying a normal pressure to the pipes to instead the role of the inside water of pipes, the loads of pipes conforms to the axial linear distribution, and the role of oriented trestles are simplified by assuming the displacement constraint of $\mathrm{X}$ and $\mathrm{Y}$ direction of pipes. The finite element models of fixing trestles are shown in Fig.1 and Fig.2.

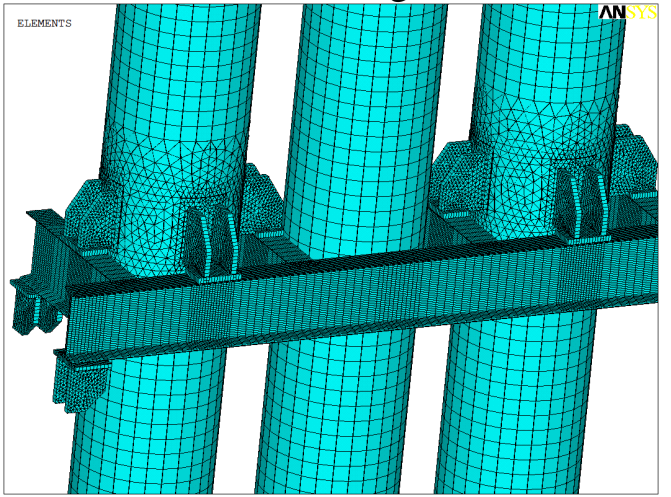

Fig.1 Fixing trestles of two sides of 3/4/4M layer

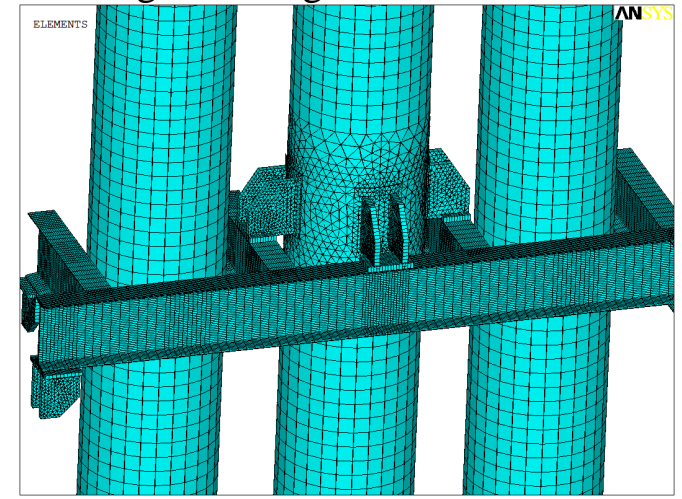

Fig.2 Fixing trestles of middle of $5 / 6$ layer

\section{Analysis}

Mode analysis. Modal analysis is used to determine vibration characteristics of the pipe system, including natural frequencies and mode shapes, which are the important parameters in the design of dynamic loads. By calculating the first 2000 natural frequencies to ensure modal participation quality of all directions of the pipe system can exceed $90 \%{ }^{[3]}$, the ten natural frequencies and modes are shown in Table 1.

Table1 Partial result of modal analysis of multilayer pipe support system

\begin{tabular}{cccc}
\hline Mode & Frequence $/ \mathrm{Hz}$ & Mode & Frequence/Hz \\
\hline 1 & 18.012 & 6 & 22.273 \\
\hline 2 & 18.644 & 7 & 22.273 \\
\hline 3 & 20.309 & 8 & 22.454 \\
\hline 4 & 22.062 & 9 & 22.454 \\
\hline 5 & 22.062 & 10 & 33.229
\end{tabular}

Response spectrum analysis. According to the requirements of the seismic response analysis norms, the equipment is usually required to run safely on the base of safe shutdown earthquake (SSE). In this paper, the response spectrum is based on seismic response spectrum of a certain factory, which was provided by China Nuclear Power Engineering Company, and the earthquake response spectrum of the floor in horizontal and vertical direction is shown in Fig.3. 

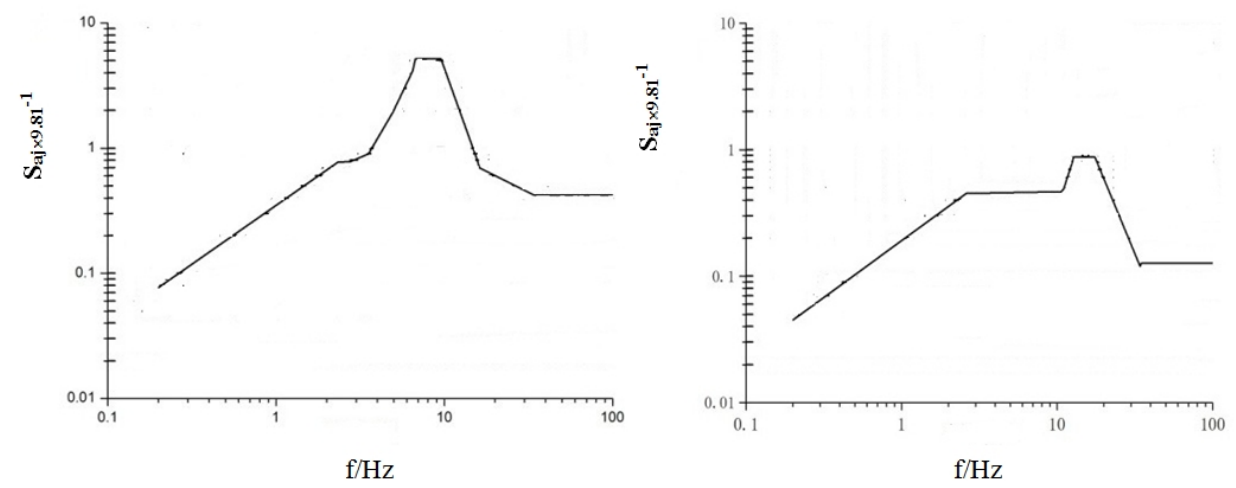

Fig.3 Earthquake response spectrum of the floor in horizontal and vertical direction

On the basis of modal analysis results, the three different dimensional displacement response can be obtained by imputing three orthogonal directional earthquake response spectrum of SSE to multi-point response spectrum, and then the stress and displacement response of the multilayer pipe support system under the combined operating conditions can be obtained by the SRSS method. The stress and displacement response of the multilayer pipe support system are shown in Fig.4 and Fig.5.

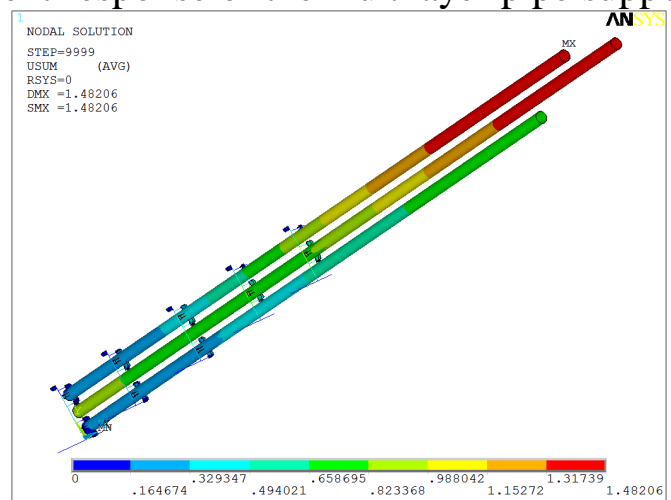

Fig.4 SSE Displacement Response o System

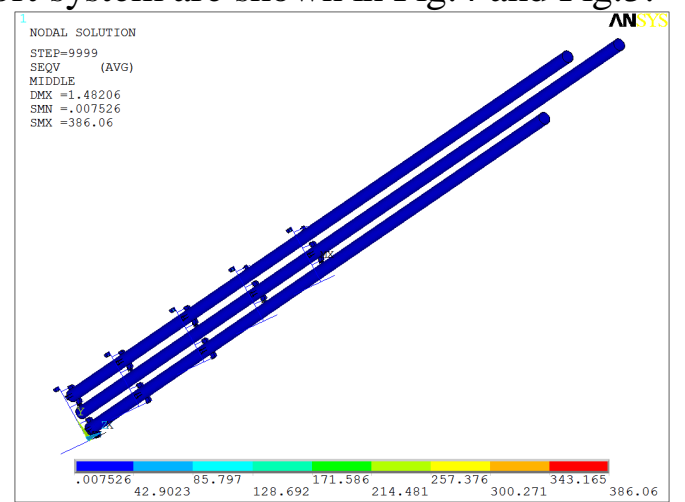

Fig.5 SSE Stress Response of System

Fig.5 shows that the maximum stress $386.1 \mathrm{Mpa}$ of the multilayer pipe support system appears at the end of the sixth layer, which is due to the structural discontinuities in connected parts of ear-type bearing and beam, and the result of some local stresses concentrating is normal and inevitable. The stress evaluation of the plate and shell structures are shown in Table 2.

Table 2 Stress evaluation of the plate and shell structures

\begin{tabular}{cccc}
\hline Degree & load combinations & components & strength evaluation \\
\hline $\mathrm{D}$ & pressure+ gravity+ SSE & $\begin{array}{c}\text { the plate and shell } \\
\text { structures }\end{array}$ & $\sigma_{m} \leq 1.5 \mathrm{~S}, \sigma_{m}+\sigma_{b} \leq 2.25 \mathrm{~S}$ \\
\hline
\end{tabular}

For the strength evaluation of the fixing trestles, Fig.5 shows that the stress results of pipes is less than the stress results of the fixing trestles, so it just need to evaluate the stress results of the fixing trestles according to the same criteria. Fig. 6 and Fig. 7 show the stress results of the SSE and two kinds of stress combinations ${ }^{[4]}$.

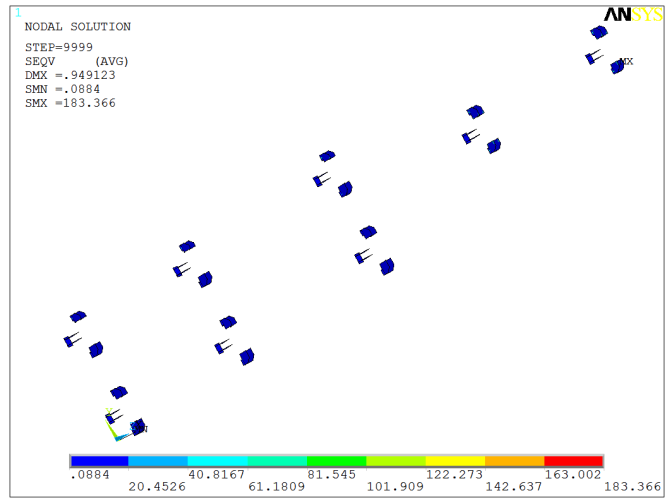

Fig.6 SSE Membrane Plus Bending Stress

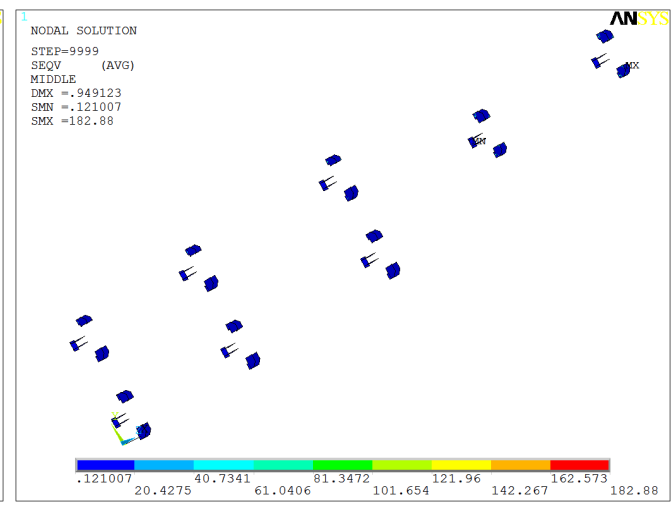

Fig.7 SSE Membrane Stress 
From the above results and the evaluation criteria ${ }^{[4]}$, the combined stress of maximum membrane stress and maximum bending stress of the fixing trestles is $183.4 \mathrm{MPa}$, and the single maximum membrane stress is $182.9 \mathrm{Mpa}$, which occurred in the bottom of the plate, showing that two kinds of combined stress meet the strength criteria.

For the stiffness evaluation of the fixing trestles, according to the results of finite element calculation, the above Fig. 4 shows that the maximum displacement of the fixing trestles is less than the maximum displacement of pipes, so it just need to evaluate the displacement results of pipes according to the same criteria. The allowed deformation limit of the equipment in the design of degree $\mathrm{D}$ is $d_{a / l} \leq 0.9 d_{\max }$ based on the corresponding seismic analysis criteria, and $d_{\max }$ is the allowed maximum deformation, which avoid the equipment from being damaged. Without any specification, the common approach is to take the length of $1.5 \%$ of pipes as the maximum allowable deformation limits ${ }^{[5]}$, on the basis of the HVAC implementation norms. As shown in Fig.8, the maximum displacement of pipes is $1.48 \mathrm{~mm}$, which occurred in the top of pipes, showing that the displacement of the plate and shell structures meets the stiffness criteria.

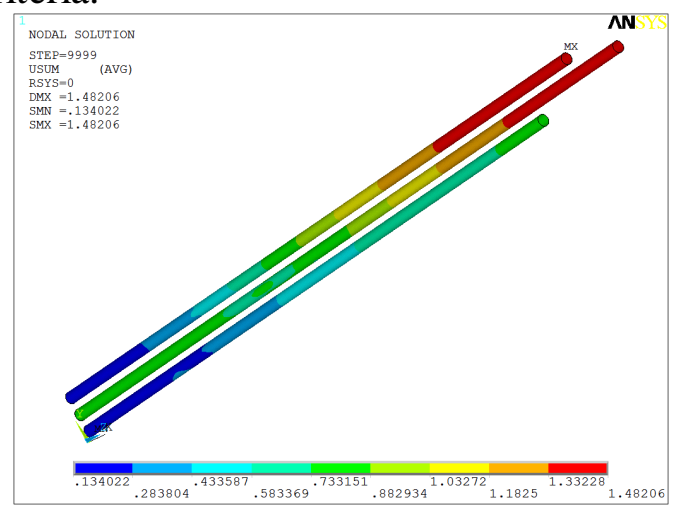

Fig.8 SSE: Stress Response of Pipes

\section{Conclusion}

In this paper, the results indicate that the strength and stiffness of fixing trestles and pipes satisfy the requirements based on both spectrum analysis and static analysis. For real operating conditions, combined loads of the multilayer pipe support system also include the case of other loads, such as wind load excitation, dynamic role of other pipeline components, and water hammer effect, so it is necessary to consider more comprehensive and dynamic analysis for designing the most favorable seismic measures.

\section{References}

[1] Casarotti chiara: Journal of Earthquake Engineering and Engineering Vibration, Vol. 5 (2008), p.119.

[2] A. Zona, M. Barbato, J. Conte: Journal of Structure Engineering, Vol. 134(2008), p.986.

[3] H.H. Wang, Z.P. Xiong, C. Liu, P. Li and Y. Wang: Journal of East China University of Science and Technology, Vol. 3 (20114), p.394 (In Chinese).

[4] W.X. Zhou, J.G. Zhang, D.Z. Wang: Journal of Atomic Energy Science and Technology, Vol. 45(2011), p.54 (In Chinese).

[5] M.X. OU, L. Zhao, W.D. Shi and F. Tian: Journal of Nuclear Power Engineering, Vol. 34(2013), p.133 (In Chinese). 